\title{
EFFECT OF THERMAL DISTORTION ON PERFORMANCE OF HYBRID JOURNAL BEARING
}

\author{
Deepam Goyal ${ }^{\mathrm{a}}$, Nitesh Parmar ${ }^{\mathrm{a}}$, Prateek Srivastava ${ }^{\mathrm{a}}$, Rajeev Kumar Dang ${ }^{\mathrm{b}}$, \\ V. Senthil ${ }^{c}$, Vijay Kumar ${ }^{*}$ \\ ${ }^{a}$ Chitkara University Institute of Engineering and Technology, Chitkara University, Punjab, \\ India \\ ${ }^{b}$ Department of Mechanical Engineering, UIET, PUSSGRC, Hoshiarpur, Punjab, India \\ ${ }^{c}$ Department of Mechanical Engineering, Chandigarh College of Engineering and Technology, \\ Chandigarh, India. \\ D.O.I- 10.51201/12242 \\ https://doi.org/10.51201/12242
}

\begin{abstract}
This paper presents static and dynamic performance characteristics for a hole-entry hybrid journal bearing system by applying theoretical investigation. The oil-film thickness was affected by the bush deformation. Temperature change and deformation because of oil-film pressure are the main contributors toward the total deformation in bearing bush. This work intends to investigate the relative influence of each of these components on performance characteristics. The journal temperature was calculated by use of oil-film temperature. Finite element method was applied for solving the governing equations by using appropriate iterative technique and for computation of journal temperature. Study was performed on symmetric and asymmetric hole-entry hybrid journal bearing configurations to evaluate the thermoelastohydrostatic performance for constant flow valve flow restrictor. Performance of the hole-entry hybrid journal bearing shows that the selection of coefficient of thermal deformation plays vital role in controlling the variation in key bearing design parameters as compared to change in temperature of the lubricating oil and bush.
\end{abstract}

KEYWORDS: Hole-Entry, Thermoelastohydrostatic, Hybrid Bearing

\section{INTRODUCTION}

Thermoelastic deformation of bush is caused by the increase in temperature and generation of hydrodynamic pressure in journal bearing [1,2]. These deformations impact the bearing performance characteristics and alter the shape of the lubricant film profile. Gethin [3] carried out a theoretical thermoelastohydrodynamic (TEHD) study by applying finite element method for a journal bearing in which oil had been supplied by two axial grooves. Elastic deformation of the bush was approximately computed by using the bearing centerline pressure and by using parabolic shaped pressure distribution in axial 
direction. It was concluded that the bearing deformation considered being important parameter in the thermal studies of bearings having a thick film, high thickness of bush wall with high aspect ratio. Recently, Linjamaa et al. [4] numerically computed the performance of journal bearing using multi-physical model. It was found that performance of journal bearings is affected by elastic and thermal deformations with characteristics of polymer layered material causing significant impact on quantum of deformation. Hybrid bearings need to be strictly designed and manufactured as per operating conditions due to their sensitivity towards such parameters which affect their performance. Mongkolwongrojn and Aiumpronsin [5] investigated the TEHD performance of fluid film bearing by analyzing the cumulative impact of roughness on bearing surface and lubricant behaviour (non-Newtonian). Hybrid model was used by incorporating Carreau viscosity model in modified Reynolds equation, journal bearing's stability region was increased using higher power law exponent at extreme condition of TEHD lubrication.

Sinhasan and Chandrawat [6] examined the elastic deformation of a journal bearing, which was designed with two axial grooves for oil supply and using a flexible shell in a rigid housing. Khonsari and Wang [7] studied the performance of fluid film bearing by including the impact on shaft and bush by thermoelastic deformation and resultant TEHD model was showing close match with experimental results in term of bearing performance. Journal bearings are normally double layered, which are constructed by using softer material on the inner surface and harder material such as steel is enveloped around babbit material. Khonsari and Wang [8] used their thermal model developed in [7] for the investigation of thermal effects of double layered bearings. Result showed the maximum temperature of pad surface was $4^{\circ} \mathrm{C}$ higher compared to a single material bearing. More thicker babbit layer on the bearing surface will show higher surface temperature. Hagemann and Schwarze [9] theoretically and experimentally analyzed the journal bearings of type direct lubricated tilting pad coupled leading edge grooves (LEG). Comprehensive code was written for novel LEG model and results were validated using test data of pivot oriented tilted bearing having four pads. Experimental and theoretical results were in close proximity in the investigated operating range, due to consideration of accurate boundary conditions.

Yang and Palazzolo [10] analyzed tilted pad bearing by applying computational fluid dynamics (CFD) technique and developing finite element based model for prediction of dynamic coefficients, heat transfer, elastic deflection and fluid dynamics. By watching 
the journal and pad movements around the operating point, stiffness and damping coefficients were found to calculate the variation in forces and moments. Developed code of advanced 3-D Reynolds equation by considering thermal effects validated the CFD model for the case of ignored flow in between pad are and boundary conditions were provided as identical for CFD and Reynolds model. Result showed good agreement between experimental and validation case. Vinh et al. [11] analyzed performance of the circular journal bearings running at very slow speed of $60 \mathrm{rpm}$ and at high loads. It was reported that due to existence of partial lubrication, exact sized bearing cannot be designed with thermohydrodynamic (THD) model, and instead TEHD model correctly predicts bearing dimensions. El-Butch and Ashour [12] introduced distortion of the elastic pad due to temperature of the pad. Results showed that there was increase in value of minimum fluid film thickness with elastic pad in comparison to rigid pad. Use of rubber pad does not significantly affect the load carrying capacity and maximum pressure of the bearing. Selfadjusting properties of the rubber pad bearing enables to achieve highest minimum fluid film thickness which leads to safe running without having direct contact. Most recently, the work published by Pinedo et al. [13] studied heating phenomenon developed due to friction between seal surface of thermoplastic polyurethane and nitrile butadiene rubber during operation. Study of different operating parameters, namely, sliding velocity, material used for sealing, steel surface conditions and applied load was done. Comparison of experimental outcomes was performed with results of analytical models, to identify the advantages and limitations of the latter.

In past hydrostatic journal bearings were only used for supporting higher loads at standstill conditions or slow speeds and lower eccentricities were needed for precision machine tools. But, in last couple of decades, industries requirement of high speed application has created the need for design modification and performance improvement of hydrostatic bearings. Meeting such stringent requirements of industry, alternative bearing design and configuration is required to be developed. For meeting high speed and heavy loading operation condition, hybrid journal bearings were developed and tested on real time machine operation. Hybrid journal bearings were developed by incorporation of characteristics and mechanisms of hydrostatic and hydrodynamic bearings.

The hybrid journal bearing configurations are of basically two types i.e. recessed and non-recessed. In the conventional recessed hydrostatic/hybrid journal bearings, major portion of the bearing land gets occupied by the recesses and thus, for speedy operations, significant hydrodynamic action is not generated. For these conditions, the non-recessed 
type of hybrid journal bearings were found to perform better and proved more appropriate [14] and hence they can be used as an alternatives for recessed bearings. These classes of bearings when designed appropriately perform superior to multi recessed bearing with the appropriate characteristics of hydrodynamic bearings at high speed and hydrostatic bearings at low speed. A thorough literature review [15-17] on the hole-entry hydrostatic as well as hybrid journal bearings emphasize that most of the studies reported in the literature neglected the thermal effects in the analysis. Thus, the current work focusses to analyze the key indicators related to hole-entry hybrid journal bearing by analyzing the change in viscosity with respect to increase in lubricant temperature and give inputs to bearing designers.

\section{FLUID FILM PRESSURE}

Reynold's equation has been applied for computing the oil film pressure generated in the clearance region by assuming incompressible fluid flow and equation 1 shows the nondimensional form of Reynolds equation.

$$
\frac{\partial}{\partial \alpha}\left(\bar{h}^{3} \bar{F}_{2} \frac{\partial \bar{p}}{\partial \alpha}\right)+\frac{\partial}{\partial \beta}\left(\bar{h}^{3} \bar{F}_{2} \frac{\partial \bar{p}}{\partial \beta}\right)=\Omega\left[\frac{\partial}{\partial \alpha}\left\{\left(1-\frac{\bar{F}_{1}}{\bar{F}_{o}}\right) \bar{h}\right\}+\frac{\partial \bar{h}}{\partial \bar{t}}\right.
$$

where, $\bar{h}$ is dimensionless oil film thickness; $\Omega$ is speed parameter; $\bar{F}_{0}, \bar{F}_{1}$, and $\bar{F}_{2}$ are nondimensional viscosity function; $\alpha, \beta$ are circumferential and axial coordinates can be defined as

$$
\begin{aligned}
& \bar{F}_{0}=\int_{0}^{1}(1 / \bar{\mu}) d \bar{z}, \quad \bar{F}_{1}=\int_{0}^{1}(\bar{z} / \bar{\mu}) d \bar{z}, \quad \text { and } \\
& \bar{F}_{2}=\int_{0}^{1}(\bar{z} / \bar{\mu})\left\{\bar{z}-\left(\bar{F}_{1} / \bar{F}_{o}\right)\right\} d \bar{z}
\end{aligned}
$$

In above equation of $\bar{F}_{0}$, and $\bar{F}_{2}, \bar{\mu}$ is non-dimensional viscosity, which is temperature dependent and computed by the relation [6].

$$
\bar{\mu}=\exp \left[\bar{a}_{1}\left[\frac{1+273.12 / T_{r}}{\bar{T}_{f}+273.12 / T_{r}}-1\right]\right]
$$

\section{Boundary conditions}

For the evaluation of lubricating oil flow field, following boundary conditions are described:

a) Pressure is zero for nodes present at external boundary of bearing; $\left.\bar{p}\right|_{\beta=\mp 1.0}=0.0$. 
b) Pressure will be equal for nodes present on a hole.

c) At the trailing edge of positive zone; $\bar{p}=(\partial \bar{p} / \partial \alpha)=0.0$.

\section{Restrictor Flow Equation}

Flow control module i.e. restrictors were used along with externally pressurized journal bearings. In various mechanical applications, flow is controlled by various devices, such as, orifice, capillary mechanism or constant flow valve. In the present analysis, flow is controlled through capillary device and flow of lubricating oil through capillary restrictor has been written as in equation 2 .

$\bar{Q}_{R}=\bar{C}_{s 2}\left(1-\bar{p}_{c}\right)$

where, $\bar{C}_{s 2}$ is restrictor design parameter

\section{FLUID FILM TEMPERATURE}

Non-dimensional type of equation to evaluate temperature of lubricating oil film $\bar{T}_{f}$ calculated by using a three dimensional form of energy equation is provided below as equation 3.

$$
\bar{h}^{2}\left[\bar{u} \frac{\partial \bar{T}_{f}}{\partial \alpha}+\bar{v} \frac{\partial \bar{T}_{f}}{\partial \beta}+\frac{\bar{w}}{\bar{h}} \cdot \frac{\partial \bar{T}_{f}}{\partial \bar{z}}\right]=\bar{P}_{e}^{*}\left(\frac{\partial^{2} \bar{T}_{f}}{\partial \bar{z}^{2}}\right)+\bar{D}_{e} \bar{\mu}\left[\left(\frac{\partial \bar{u}}{\partial \bar{z}}\right)^{2}+\left(\frac{\partial \bar{v}}{\partial \bar{z}}\right)^{2}\right]
$$

Where, $\bar{u}, \bar{v}, \bar{w}$ are dimensionless velocity components in $\mathrm{x}, \mathrm{y}$ and $\mathrm{z}$ directions respectively; $\bar{P}_{e}^{*}$ is inverse Peclet number; $\bar{D}_{e}$ is dissipation number

\section{TEMPRATURE DISTRIBUTION IN BEARING}

Equation 4 shows the equation for heat conduction and written in cylindrical co-ordinates and used to calculate fluid-film temperature at bush interface:

$$
\frac{1}{\bar{r}} \frac{\partial}{\partial \bar{r}}\left(\bar{k}_{b} \bar{r} \frac{\partial \bar{T}_{b}}{\partial \bar{r}}\right)+\frac{1}{\bar{r}^{2}} \frac{\partial}{\partial \alpha}\left(\bar{k}_{b} \frac{\partial \bar{T}_{b}}{\partial \alpha}\right)+\bar{r} \frac{\partial}{\partial \beta}\left(\bar{k}_{b} \frac{\partial \bar{T}_{b}}{\partial \beta}\right)=0
$$

where, $\overline{k_{b}}$ is dimensionless thermal conductivity of bush

Discretization of the lubricating oil flow field has been done by use of 8 node element of hexahedral linear isoparametric type. The 3-D finite element meshing for performing thermal analysis has been made compatible with the 2-D grid, which is applied for solving Reynolds equation along the axial and circumferential directions. For conducting the thermal analysis of bush of the bearing it is discretized by hexahedral isoparametric 
elements of 8 nodes. Grid developed through discretization is suitable compared to lubrication grid and for fluid domain energy equation analysis. The temperature $\bar{T}_{b}$, in an element can be evaluated using the relation:

\section{Thermal Boundary Conditions}

Equations (3) and (4) have been solved by assuming boundary conditions, which have been expressed in table 1 .

Table 1: Thermal boundary conditions

\begin{tabular}{|c|l|c|}
\hline S.No. & \multicolumn{1}{|c|}{ Location } & \multicolumn{1}{|c|}{ Boundary condition } \\
\hline 1 & At interface of journal and oil & $\left.\bar{T}_{f}\right|_{\bar{z}=1}=\bar{T}_{J}$ \\
\hline 2 & At interface of bush and oil & $\left.\bar{T}_{f}\right|_{\bar{z}=0}=\left.\bar{T}_{b}\right|_{\bar{r}=\bar{R}_{1}}$ \\
\hline 3 & $\begin{array}{l}\text { At the interface of bush for } \\
\text { maintaining continuity of heat } \\
\text { flux i.e. solid domain }\end{array}$ & $-\left.\frac{k_{b}}{R_{J}} \frac{\partial \bar{T}_{b}}{\partial \bar{r}}\right|_{\bar{r}=\bar{R}_{1}}=\left.\frac{k_{f}}{c \bar{h}} \frac{\partial \bar{T}_{f}}{\partial \bar{z}}\right|_{\bar{z}=0}$ \\
\hline 4 & $\begin{array}{l}\text { At the external face of the } \\
\text { housing }\left(\bar{r}=\bar{R}_{2}\right), \text { the free } \\
\text { convection and radiation } \\
\text { hypothesis }\end{array}$ & $\left(\partial \bar{T}_{b} / \partial \bar{r}\right)_{\bar{r}=\bar{R}_{2}}=\left(h_{b} R_{J} / k_{b}\right)\left(\left.\bar{T}_{b}\right|_{\bar{r}=\bar{R}_{2}}-\bar{T}_{a}\right)$ \\
\hline 5 & $\begin{array}{l}\text { On the lateral faces of bearing } \\
\text { hypothesis }\end{array}$ & $\left(\partial \bar{T}_{b} / \partial \beta\right)_{\bar{r}=\bar{R}_{2}}=-\left(h_{b} R_{J} / k_{b}\right)\left(\left.\bar{T}_{b}\right|_{\beta= \pm \lambda}-\bar{T}_{a}\right)$ \\
\hline 6 & \begin{tabular}{l} 
Inlet of supply holes \\
\hline
\end{tabular} & $\bar{T}_{b}=\bar{T}_{s}$ \\
\hline
\end{tabular}

\section{THERMOELASTIC DEFORMATION}

Solution of elasticity equations has been carried out for analyzing the thermal deformation and elastic behavior of bearing shell. For this, a finite length cylindrical shaped structure was considered for analysis. With the finite element based modeling and use of linear elasticity equation and principle of virtual work, the deformation in an elastic continuum is computed using system equation. For oil film thickness modification requires a radial component at the interface of oil-film and bush. In this work, journal deformation due to fluid-film pressure was neglected because in general, rigidity of journal is always higher than bush. Discretized elastic continuum system equation is defined as:

$$
[\bar{K}]\{\bar{\delta}\}=\bar{C}_{d}\left\{\bar{F}_{\Gamma}\right\}+\bar{C}_{t}\left\{\bar{F}_{T}\right\}
$$


where $\bar{C}_{d}$ (elastic deformation coefficient) $=\left(\frac{p_{s} R_{J}}{E_{b} c}\right) ;\{\bar{\delta}\}$ is displacement vector; $\bar{C}_{t}$ (thermal deformation coefficient $)=\left(\frac{\alpha_{b} T_{r} R_{J}}{c}\right)$

Therefore, matrices of $e^{\text {th }}$ element can be written as:

$$
\begin{gathered}
{[\bar{K}]^{e}=\int_{\Omega^{e}} \bar{B}^{T} \bar{D} \bar{B} \bar{r} d \Omega^{e}} \\
\left\{\bar{F}_{\Gamma}\right\}^{e}=\int_{\Gamma^{e}} N^{T} \bar{p} \bar{r} d \Gamma^{e} \\
\left\{\bar{F}_{T}\right\}^{e}=\int_{\Omega^{e}} \bar{B}^{T} \bar{D} \bar{\varepsilon}_{0} \bar{r} d \Omega^{e}
\end{gathered}
$$

where, $[\bar{B}]$ is strain matrix; $[N]$ is shape function matrix; $\Gamma$ is solution boundary; $\left\{\bar{F}_{T}\right\}$ is thermal load vector; $\left\{\bar{F}_{\Gamma}\right\}$ is traction force vector

\section{Boundary conditions}

A finite length 3D cylindrical structure affixed in a rigid housing was considered as bearing shell. Material of bearing shell has much more flexibility compared to housing.

Assumption of zero displacement was considered on the bush-housing node.

$\{\bar{\delta}\}=\left\{\begin{array}{lll}0 & 0 & 0\end{array}\right\}^{T}$

\section{Oil film thickness}

When journal bearing is under loading condition, there is requirement of minimum oil film thickness, which would avoid metal contact and friction in the bearing. Fig. 1 explains the schematic diagram of two different types of hole-entry journal bearing configurations, which shows that the oil film thickness can be varied by deformation of journal due to thermal affects and thermoelastic deformation of bearing. Oil film thickness of rigid journal bearing is shown below:

$$
\bar{h}=\bar{h}_{0}+\Delta \bar{h}
$$

where $\bar{h}_{0}$ represents the thickness of oil film under static equilibrium position of journal and is shown as

$$
\bar{h}_{0}=1-\bar{X}_{J} \cos \alpha-\bar{Z}_{J} \sin \alpha
$$

$\Delta \bar{h}$ represents the perturbation caused by dynamic conditions on the thickness of oil film. 
Modified film thickness including bearing flexibility and thermal affects is shown below:

$$
\bar{h}=\bar{h}_{0}+\Delta \bar{h}+\bar{\delta}_{f b}^{p}-\bar{\delta}_{f b}^{T}-\bar{\delta}_{J}^{T}
$$

where $\bar{\delta}_{J}^{T}$ expresses the radial deformation of journal caused by increase of journal temperature, while, $\bar{\delta}_{f b}^{p}$ and $\bar{\delta}_{f b}^{T}$ represent the radial deformations of bush because of oilfilm pressure and increase in temperature of bush respectively at the interface of oil film. All these values have been given in dimensionless form for ensuring compatibility with base equation of film thickness.

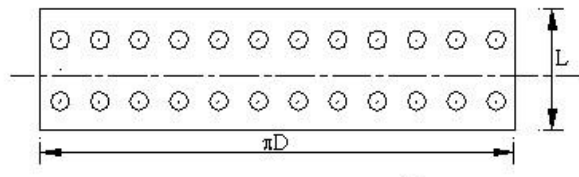

(a)

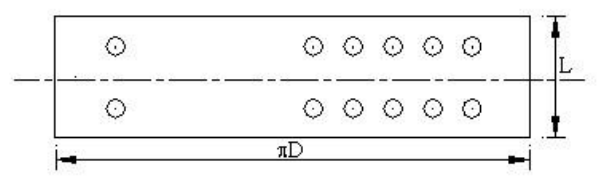

(b)
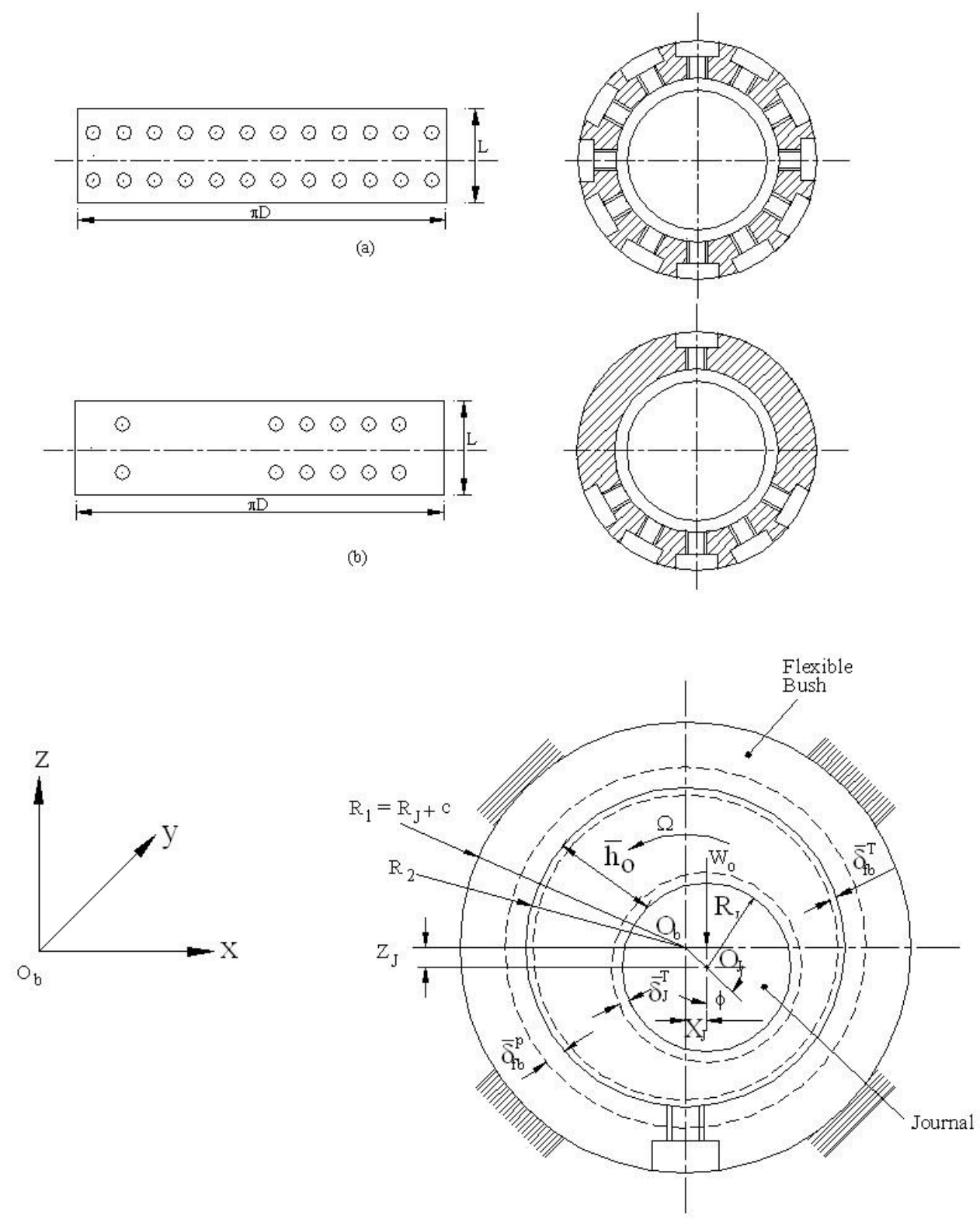

Figure 1: Hole-entry journal bearing configurations (a) symmetric (b) asymmetric (c) geometrical details and representation of thermoelastic deformation. 
Dowson et al. [18] experimental analysis showed that there is almost negligible variation of temperature along circumferential direction of journal. As a result, journal can be assumed as an isothermal object in bearing assembly. It can be stated as assumption i.e. journal has uniform temperature and is in form of axisymmetric body. Displacement of journal due to thermal affects is dependent on coefficient of linear thermal expansion and can be written as.

$\bar{\delta}_{J}^{T}=\alpha_{J} T_{r}\left(\frac{1}{\bar{c}}\right)\left(\bar{T}_{J}-1\right)$

\section{SOLUTION PROCEDURE}

Iterative numerical solution technique is used in the present work to create temperature and pressure fields in the lubricating oil film. TEHD solution of a hole-entry hybrid journal bearing requires that Reynolds, energy, elasticity and conduction equations need to be simultaneously by assuming required boundary conditions.

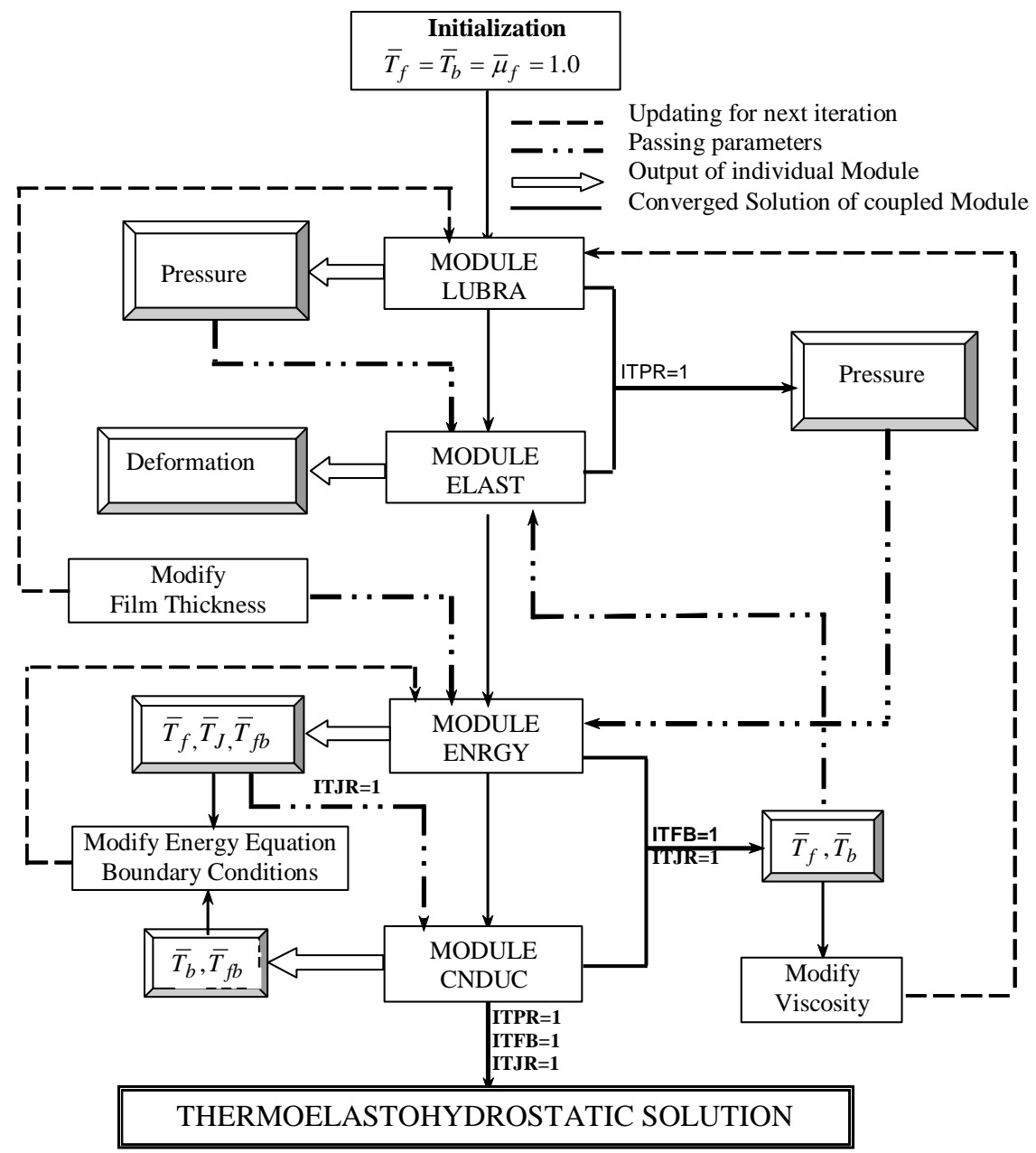

Figure 2: Solution scheme 
Figure 2 shows the combined methodology of the four modules used for obtaining the solution which were named as LUBRA, ELAST, ENRGY, CNDUC and used to develop the solution scheme. Different modules were used to solve different objectives. A fluidfilm pressure was obtained by solving the lubricant flow field equation using moduleLUBRA. Bush nodal thermoelastic deformation was evaluated by module-ELAST, it uses fluid-film nodal pressure values for computing Bush nodal thermoelastic deformation. Thereafter energy equation was solved by module-ENRGY using the converged solution of Module- ELAST and LUBRA. Evaluation of journal temperature was done from oil film temperatures by using solution obtained from energy equation. Multiple iterations for solving energy equation with modification in fluid-film journal interface boundary conditions were done, iterations were terminated until index ITJR arrives unity value. Temperature of the bush was evaluated by using module-CNDUC. Attainment of unity value of index ITFB signifies the convergence of the oil-bush interface and temperature of bush surface. Module-LUBRA is applied for obtaining new oil-film pressure field as change in temperature profile modifies the oil-film viscosity field. Iterations were repeated until convergence is achieved for oil film pressure field (ITPR=1).

\section{RESULTS AND DISCUSSION}

The total deformation in bearing is contributed by two components; deformation caused by pressure of oil film and variation in temperature. This work intends to investigate the relative influence of each of these components on performance characteristics. The parameter $\bar{C}_{t}$ as defined earlier is a measure of thermal deformation in the bearing. The variation in the static and dynamic performance characteristics for symmetric as well as asymmetric hole-entry type of journal bearings against the thermal deformation coefficient $\left(\bar{C}_{t}\right)$ are presented to see the effect of thermal deformation in the bearing bush made of different materials. The values of $\bar{C}_{t}=0.0$ and $\bar{C}_{d}=0.0$ are used for the cases in which thermal deformation and elastic deformation are to be neglected respectively. The performance characteristics obtained for $\bar{C}_{t}=0.0 ; \bar{C}_{d}=0.0$ are taken as a reference and are compared with performance characteristics computed accounting elastic and thermal deformations $\left(\bar{C}_{t} \neq 0.0 ; \bar{C}_{d} \neq 0.0\right)$. The comparison for these characteristics are presented for external load $\left(\bar{W}_{o}=1.5\right)$, speed parameter $(\Omega=1.0)$, restrictor flow $\left(\bar{Q}_{c}=0.0647\right)$, 
elastic deformation coefficient $\left(\bar{C}_{d}=0.0,1.0\right.$ and 5.0), inverse Peclet number $\left(\bar{P}_{e}^{*}=0.0855\right)$ and dissipation number $\left(\bar{D}_{e}=0.13023\right)$.

For establishing the validity of developed model, computation of mid plane temperature was carried out at different circumferential coordinates and comparison was made with already existing theoretical and experimental findings [7]. Outcomes achieved from present work were in close proximity to earlier available results and have been shown in Fig. 3.

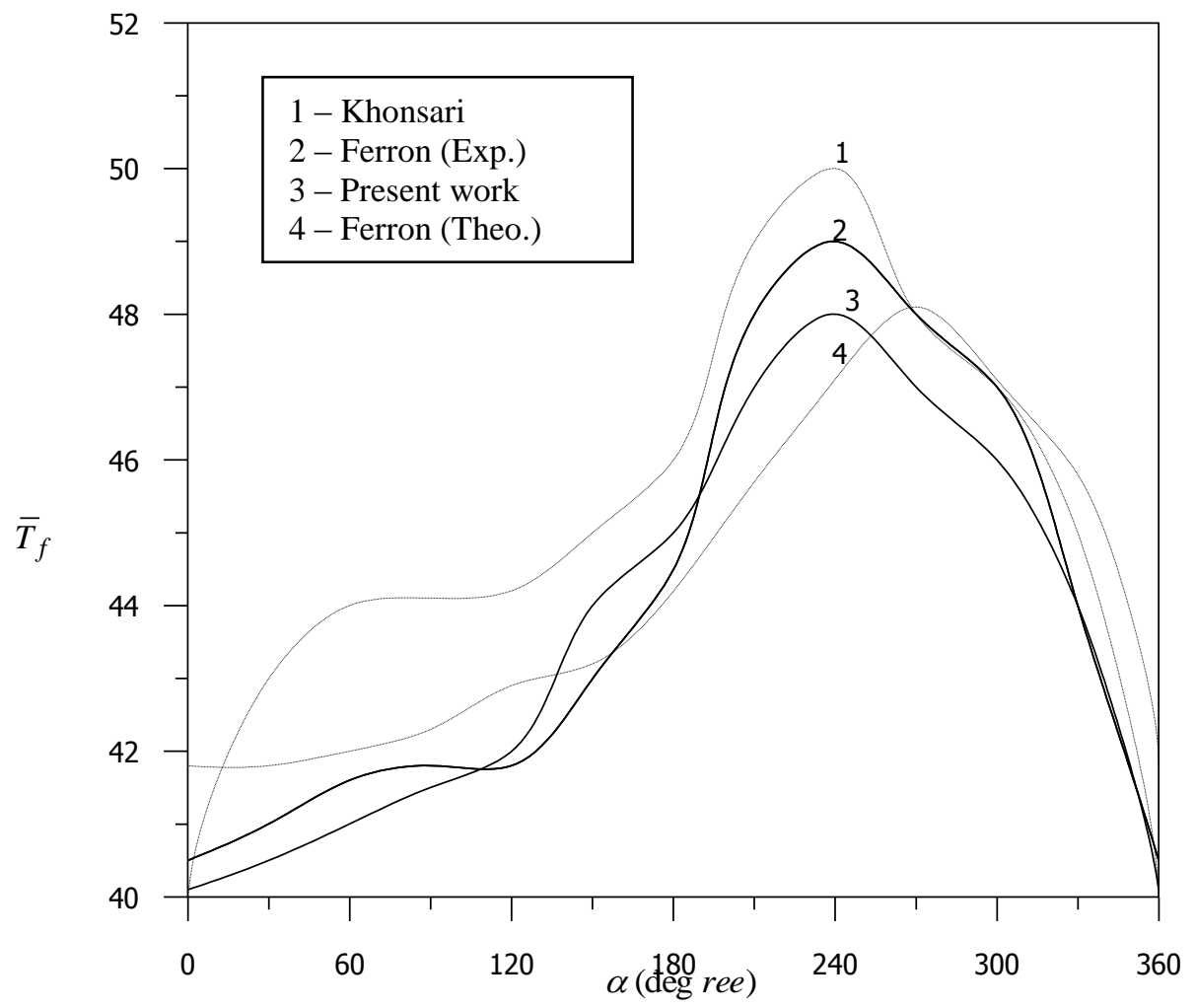

Figure 3: Variation of mid film temperature with $\alpha$

The results of simulation for investigating the effect of thermal deformation coefficient $\left(\bar{C}_{t}\right)$ on maximum pressure $\left(\bar{p}_{\max }\right)$ and minimum film thickness $\left(\bar{h}_{\text {min }}\right)$ are tabulated in Table 1. It has been noticed that the value of maximum pressure reduces with rise in $\bar{C}_{t}$ for flexible bearings $\left(\bar{C}_{d} \neq 0\right)$. This effect is more for symmetric configuration in comparison to the asymmetric. Value of $\bar{p}_{\max }$ reduces more for higher value of $\bar{C}_{t}$ and it is found to be of the order of about $18 \%\left(\bar{C}_{t}=1.25\right.$ and $\left.\bar{C}_{d}=5.0\right)$ as shown in Table 1. 
Table 1: Percentage change in static performance characteristics

\begin{tabular}{|c|c|c|c|c|c|c|c|c|c|c|c|c|c|}
\hline & \multirow{2}{*}{$\bar{C}_{t}$} & \multicolumn{2}{|c|}{$\bar{C}_{d}=0.0$} & \multicolumn{2}{|c|}{$\bar{C}_{d}=1.0$} & \multicolumn{2}{|c|}{$\bar{C}_{d}=5.0$} & \multicolumn{2}{|c|}{$\bar{C}_{d}=0.0$} & \multicolumn{2}{|c|}{$\bar{C}_{d}=1.0$} & \multicolumn{2}{|c|}{$\bar{C}_{d}=5.0$} \\
\hline & & THS & \%Diff & TEHS & \%Diff & TEHS & \%Diff & THS & \%Diff & TEHS & \%Diff & TEHS & \%Diff \\
\hline \multirow{4}{*}{$\bar{p}_{\max }$} & 0.00 & 1.019 & 0.00 & 0.980 & -3.83 & 0.875 & -14.1 & 0.728 & 0.00 & 0.724 & -0.5 & 0.710 & -2.5 \\
\hline & 0.50 & 1.018 & -0.10 & 0.953 & -6.48 & 0.859 & -15.7 & 0.730 & 0.27 & 0.718 & $\begin{array}{l}-1.4 \\
\end{array}$ & 0.707 & -2.9 \\
\hline & 0.75 & 1.017 & -0.20 & 0.940 & -7.75 & 0.851 & -16.5 & 0.731 & 0.41 & 0.716 & -1.6 & 0.705 & -3.2 \\
\hline & 1.25 & 1.017 & -0.20 & 0.916 & -10.11 & 0.836 & -18.0 & 0.731 & 0.41 & 0.713 & -2.1 & 0.703 & -3.4 \\
\hline \multirow{4}{*}{$\bar{h}_{\min }$} & 0.00 & 0.825 & 0.00 & 0.800 & -3.03 & 0.691 & -16.2 & 0.877 & 0.00 & 0.859 & -2.1 & 0.758 & -13.6 \\
\hline & 0.50 & 0.824 & -0.12 & 0.823 & -0.24 & 0.714 & -13.5 & 0.874 & -0.34 & 0.873 & -0.5 & 0.778 & -11.3 \\
\hline & 0.75 & 0.824 & -0.12 & 0.834 & 1.09 & 0.725 & -12.1 & 0.873 & -0.46 & 0.866 & -1.3 & 0.787 & -10.3 \\
\hline & 1.25 & 0.824 & -0.12 & 0.842 & 2.06 & 0.748 & -9.3 & 0.872 & -0.57 & 0.840 & -4.2 & 0.805 & -8.2 \\
\hline
\end{tabular}

It shows that the effect of $\bar{C}_{t}$ on $\bar{p}_{\max }$ and $\bar{h}_{\min }$ is almost insignificant when elastic deformation due to pressure is neglected $\left(\bar{C}_{d}=0.0\right)$. The symmetric bearing configuration is found to maintain low value of minimum oil-film thickness in comparison to asymmetric bearing. The reduction in $\bar{h}_{\min }$ for $\bar{C}_{d}=5.0$, is decreased from $16.2 \%$ at $\bar{C}_{t}=0.0$ to $9.3 \%$ at $\bar{C}_{t}=1.25$ for symmetric hole-entry bearing configuration. It implies that the thermal effects can be controlled a bit by selecting materials with higher $\bar{C}_{t}$.

The variation of dynamic performance characteristics with thermal deformation coefficient $\left(\bar{C}_{t}\right)$ is tabulated in Table 2 .

Table 2: Percentage change in dynamic performance characteristics

\begin{tabular}{|c|c|c|c|c|c|c|c|c|c|c|c|c|c|}
\hline & \multirow{2}{*}{$\bar{C}_{t}$} & \multicolumn{2}{|c|}{$\bar{C}_{d}=0.0$} & \multicolumn{2}{|c|}{$\bar{C}_{d}=1.0$} & \multicolumn{2}{|c|}{$\bar{C}_{d}=5.0$} & \multicolumn{2}{|c|}{$\bar{C}_{d}=0.0$} & \multicolumn{2}{|c|}{$\bar{C}_{d}=1.0$} & \multicolumn{2}{|c|}{$\bar{C}_{d}=5.0$} \\
\hline & & THS & \%Diff & TEHS & \%Diff & TEHS & \%Diff & THS & \%Diff & TEHS & \%Diff & TEHS & \%Diff \\
\hline \multirow{4}{*}{$\bar{S}_{z z}$} & 0.00 & 6.18 & 0.00 & 5.64 & -8.74 & 4.25 & -31.2 & 2.79 & 0.00 & 2.75 & -1.43 & 2.57 & -7.89 \\
\hline & 0.50 & 6.15 & -0.49 & 5.30 & -14.24 & 4.07 & -34.1 & 2.79 & 0.00 & 2.59 & -7.17 & 2.47 & -11.47 \\
\hline & 0.75 & 6.14 & -0.65 & 5.14 & -16.83 & 3.99 & -35.4 & 2.79 & 0.00 & 2.52 & -9.68 & 2.43 & -12.90 \\
\hline & 1.25 & 6.13 & -0.81 & 4.85 & -21.52 & 3.83 & -38.0 & 2.79 & 0.00 & 2.51 & -10.04 & 2.35 & -15.77 \\
\hline \multirow{4}{*}{$\bar{C}_{z z}$} & 0.00 & 12.91 & 0.00 & 11.97 & -7.28 & 9.54 & -26.10 & 10.20 & 0.00 & 9.85 & -3.43 & 8.69 & -14.80 \\
\hline & 0.50 & 12.83 & -0.62 & 11.34 & -12.16 & 9.18 & -28.89 & 10.10 & -0.98 & 8.99 & -11.86 & 8.06 & -20.98 \\
\hline & 0.75 & 12.81 & $\begin{array}{l}-0.77 \\
\end{array}$ & 11.06 & -14.33 & 9.01 & -30.21 & 10.07 & -1.27 & 8.61 & -15.59 & 7.78 & -23.73 \\
\hline & 1.25 & 12.79 & -0.93 & 10.53 & -18.44 & 8.69 & -32.69 & 10.03 & -1.67 & 7.94 & -22.16 & 7.27 & -28.73 \\
\hline \multirow{4}{*}{$\bar{M}_{c}$} & 0.00 & 25.43 & 0.00 & 23.38 & -8.06 & 18.13 & -28.71 & 11.88 & 0.00 & 11.75 & -1.09 & 11.19 & -5.81 \\
\hline & 0.50 & 25.31 & -0.47 & 21.98 & -13.57 & 17.41 & -31.54 & 11.87 & -0.08 & 11.13 & -6.31 & 10.77 & -9.34 \\
\hline & 0.75 & 25.28 & -0.59 & 21.34 & -16.08 & 17.07 & -32.87 & 11.86 & -0.17 & 10.86 & -8.59 & 10.58 & -10.94 \\
\hline & 1.25 & 25.25 & -0.71 & 20.16 & -20.72 & 16.42 & -35.43 & 11.86 & -0.17 & 10.41 & -12.37 & 10.25 & -13.72 \\
\hline
\end{tabular}

Generally, the values of direct stiffness coefficients $\left(\bar{S}_{z z}\right)$ reduce with the increase of $\bar{C}_{t}$.

It is further observed that at a constant value of thermal deformation coefficient $\bar{S}_{z z}$ 
reduces with increasing flexibility. There is more percentage change in $\bar{S}_{z z}$ with higher elastic deformation coefficient $\left(\bar{C}_{d}=5.0\right)$ for symmetric configuration ( $38 \%$ at $\bar{C}_{t}=1.25$ ) as noticed from Table 2 and it is $15.77 \%$ at $\bar{C}_{t}=1.25$ for asymmetric bearing configuration. The variations of direct damping coefficient $\bar{C}_{z z}$ are found to decrease with an increase in thermal deformation coefficient. For symmetric configuration, a maximum reduction of $32 \%$ (at $\bar{C}_{t}=1.25$ and $\bar{C}_{d}=5.0$ ) has been observed for $\bar{C}_{z z}$ compared with the case when both elastic and thermal deformations are neglected. There has been decrease in value of $\bar{C}_{z z}$ of the order of $28.7 \%$ for asymmetric configuration. Also, reduction in critical journal mass $\left(\bar{M}_{c}\right)$ is observed with $\bar{C}_{t}$ and the reduction is less in asymmetric configuration as compared to symmetric configuration. A maximum decrease in value of $\bar{M}_{c}$ is observed, which is approximately $35.5 \%$ (at $\bar{C}_{t}=1.25$ and $\bar{C}_{d}=5.0$ ) for symmetric and $13.7 \%$ (at $\bar{C}_{t}=1.25$ and $\bar{C}_{d}=5.0$ ) for asymmetric configurations.

\section{CONCLUSION}

Static and dynamic performance characteristics for a hole-entry hybrid journal bearing system by applying theoretical investigation has been presented in this paper. Results show the reduction in magnitude of $\bar{h}_{\text {min }}$ for constant flow valve included non- recessed type of hybrid journal bearing. This enables the journal to run at higher value eccentricity ratio $(\varepsilon)$ when bearing flexibility increases for any specified value of thermal deformation coefficient $\left(\bar{C}_{t}\right)$. The reduction in the value of $\bar{h}_{\min }$ can however, be compensated by selecting a proper material for bearing having large value of $\bar{C}_{t}$. 


\section{REFERENCES}

[1] Ferron J, Frene J, Boncompain R. A study of the thermohydrodynamic performance of a plain journal bearing comparison between theory and experiments. 1982;105:1-7.

[2] Garg HC, Sharda HB, Kumar V. On the design and development of hybrid journal bearings: a review. Tribotest 2006;12:1-19. https://doi.org/10.1002/tt.1.

[3] Gethin DT. An Investigation into Plain Journal Bearing Behaviour Including Thermo-Elastic Deformation of the Bush. Proceedings of the Institution of Mechanical Engineers, Part C: Journal of Mechanical Engineering Science 1985;199:215-23. https://doi.org/10.1243/PIME_PROC_1985_199_116_02.

[4] Linjamaa A, Lehtovaara A, Larsson R, Kallio M, Söchting S. Modelling and analysis of elastic and thermal deformations of a hybrid journal bearing. Tribology International 2018;118:451-7. https://doi.org/10.1016/j.triboint.2017.02.029.

[5] Mongkolwongrojn M, Aiumpronsin C. Stability analysis of rough journal bearings under TEHL with non-Newtonian lubricants. Tribology International 2010;43:1027-34. https://doi.org/10.1016/j.triboint.2009.12.039.

[6] Sinhasan R, Chandrawat HN. Analysis of a two-axial-groove journal bearing including thermoelastohydrodynamic effects. Tribology International 1989;22:34753. https://doi.org/10.1016/0301-679X(89)90151-5.

[7] Khonsari MM, Wang SH. On the fluid-solid interaction in reference to thermoelastohydrodpamic analysis of journal bearings. Journal of Tribology 1991;113:398-404. https://doi.org/10.1115/1.2920635.

[8] Khonsari MM, Wang SH. On the maximum temperature in double-layered journal bearings. Journal of Tribology 1991;113:464-9. https://doi.org/10.1115/1.2920647.

[9] Hagemann T, Schwarze H. Theoretical and experimental analyses of directly lubricated tilting-pad journal bearings with leading edge groove. Journal of Engineering for Gas Turbines and Power 2019;141. https:// doi.org/10.1115/1.4041026.

[1] Yang J, Palazzolo A. Three-Dimensional Thermo-Elasto-Hydrodynamic Computational Fluid Dynamics Model of a Tilting Pad Journal Bearing - Part II: Dynamic Response. Journal of Tribology 2019;141. https:// doi.org/10.1115/1.4043350. 
[11] Vinh DP, Chatterton S, Pennacchi P. Static and dynamic behaviors of a cylindrical hydrodynamic journal bearing operating at very low Sommerfeld numbers. Mechanisms and Machine Science 2019;73:3835-44. https://doi.org/10.1007/9783-030-20131-9_380.

[12] El-Butch AM, Ashour NM. Analysis of heavy duty tilting-pad journal bearing taking into account pad distortion and possible adoption of rubber pad segments. Tribology International 1999;32:285-93. https://doi.org/10.1016/ S0301-679X(99)00046-8.

[13] Pinedo B, Hadfield M, Tzanakis I, Conte M, Anand M. Thermal analysis and tribological investigation on TPU and NBR elastomers applied to sealing applications. Tribology International 2018;127:24-36. https://doi.org/10.1016/ j.triboint.2018.05.032.

[14] Chasalevris AC, Nikolakopoulos PG, Papadopoulos CA. Dynamic effect of bearing wear on rotor-bearing system response. Journal of Vibration and Acoustics, Transactions of the ASME 2013;135. https://doi.org/10.1115/1.4007264.

[15] Khakse PG, Phalle VM, Mantha S. Orifice compensated performance characteristics of hybrid hole-entry conical journal bearing. Proceedings of the Institution of Mechanical Engineers, Part J: Journal of Engineering Tribology 2017;231:316-31. https://doi.org/10.1177/1350650116654803.

[16] Kushare PB, Sharma SC. Nonlinear transient stability study of two lobe symmetric hole entry worn hybrid journal bearing operating with non-Newtonian lubricant. Tribology International 2014;69:84-101. https://doi.org/10.1016/ j.triboint.2013.08.014.

[17] Lin $Q$, Wei $Z$, Wang $N$, Zhang $Y$. Effect of recess configuration on the performances of high-speed hybrid journal bearing. Industrial Lubrication and Tribology 2016;68:301-7. https://doi.org/10.1108/ILT-07-2015-0115.

[18] Dowson D, Hudson JD, Hunter B, March CN. An Experimental Investigation of the Thermal Equilibrium of Steadily Loaded Journal Bearings. Proceedings of the Institution of Mechanical Engineers, Conference Proceedings 1966;181:70-80. https://doi.org/10.1243/pime_conf_1966_181_034_02. 